\title{
Activation of Nigrostriatal Dopamine Neurons during Fear Extinction Prevents the Renewal of Fear
}

\author{
Courtney A Bouchet', Megan A Miner', Esteban C Loetz', Adam J Rosberg', Holly S Hake', \\ Caroline E Farmer', Mykola Ostrovskyy, Nathan Gray and Benjamin N Greenwood", \\ 'Department of Psychology, University of Colorado, Denver, CO, USA
}

\begin{abstract}
Manipulations that increase dopamine (DA) signaling can enhance fear extinction, but the circuits involved remain unknown. DA neurons originating in the substantia nigra (SN) projecting to the dorsal striatum (DS) are traditionally viewed in the context of motor behavior, but growing data implicate this nigrostriatal circuit in emotion. Here we investigated the role of nigrostriatal DA in fear extinction. Activation of SN DA neurons with designer $G_{q}$-coupled receptors exclusively activated by designer drugs $\left(G_{q}\right.$-DREADD) during fear extinction had no effect on fear extinction acquisition, but enhanced fear extinction memory and blocked the renewal of fear in a novel context; a pattern of data paralleled by cFos expression in the central amygdala. DI receptors in the DS are a likely target mediating the effects of SN DA activation. DI-expressing neurons in the medial DS (DMS) were recruited during fear extinction, and $G_{\mathrm{q}}$-DREADD-induced DA potentiated activity of D I-expressing neurons in both the DMS and the lateral DS (DLS). Pharmacological activation of DI receptors in the DS did not impact fear extinction acquisition or memory, but blocked fear renewal in a novel context. These data suggest that activation of SN DA neurons and DS DI receptors during fear extinction render fear extinction memory resistant to the disrupting effects of changes in contextual contingencies, perhaps by recruiting habitual learning strategies involving the DLS. Nigrostriatal DA thus represents a novel target to enhance long-term efficacy of extinction-based therapies for anxiety and trauma-related disorders.

Neuropsychopharmacology (2018) 43, 665-672; doi:I0.1038/npp.2017.235; published online 15 November 2017
\end{abstract}

\section{INTRODUCTION}

Extinction of traumatic memories is a major goal of therapeutic strategies for anxiety and trauma-related disorders, but extinction memories are labile and fear associated with traumatic memories can resurface even following successful extinction. Fear renewal is the return of conditioned responding following extinction in contexts different from that in which extinction occurred (Bouton and Ricker, 1994) and is a primary contributor to relapse (Goode and Maren, 2014). Recent work has therefore focused on the neurocircuits underlying fear renewal (Chen et al, 2017; Knapska et al, 2012), with a circuit involving ventral hippocampus, prefrontal cortex, and amygdala emerging as central to fear renewal (Wang et al, 2016; Knapska and Maren, 2009; Sotres-Bayon and Quirk, 2010). Targeting this canonical circuitry with pharmacological or behavioral tools, however, has yielded mixed results in reducing renewal (Fitzgerald et al, 2014). Identification of novel means to enhance fear extinction and prevent renewal is of utmost importance to mental health.

\footnotetext{
*Correspondence: Dr BN Greenwood, Department of Psychology, University of Colorado Denver, North Classroom Room 5005F, CB 173, PO Box 173364, Denver, CO 802 17-3364, USA, Tel: + I 303315 7626, E-mail: Benjamin.greenwood@ucdenver.edu

Received 13 May 2017; revised 23 September 2017; accepted 25 September 2017; accepted article preview online 4 October 2017
}

Dopamine (DA) is a memory modulator implicated in fear extinction (Haaker et al, 2013; Abraham et al, 2014). Recent theories proffer that fear extinction could involve learning a new association between the conditioned stimulus (CS) and a positive-affective state stemming from the relief that the predicted unconditioned stimulus (US) no longer follows the CS (Tronson et al, 2012); an association that could be supported by DA. Indeed, high-frequency (phasic) DA release in the striatum encodes both reward value (Howe et al, 2013) and prediction error (Schultz, 2016). These functions of DA are traditionally thought to involve the mesolimbic DA pathway, originating in the ventral tegmental area (VTA) and terminating in the nucleus accumbens (NAc). Consistent with this view, phasic DA release in the NAc increases during fear extinction (Badrinarayan et al, 2012), and D2 receptor signaling in the NAc has been reported to be necessary for fear extinction (Holtzman-Assif et al, 2010). Emerging data indicate that functions of the nigrostriatal DA pathway, originating in the substantia nigra (SN) and terminating in the dorsal striatum (DS), overlap with those of mesolimbic DA (Kravitz and Kreitzer, 2012a; Wise, 2009). Indeed, although nigrostriatal DA is traditionally viewed in the context of motor behavior, recent data reveal a role for the DS, particularly DS D1expressing neurons preferentially responsive to phasic DA release (Dreyer et al, 2010), in reinforcement (Kravitz et al, 2012b) and emotional behavior (Lenz and Lobo, 2013). Despite potential involvement of multiple DA pathways in 
fear extinction, the effects of direct manipulations of nigrostriatal DA on fear extinction and renewal have yet to be investigated.

The goal of the current experiments was to begin to elucidate the role of nigrostriatal DA in fear extinction and renewal. Viral-mediated expression of designer $\mathrm{G}_{\mathrm{q}}$-coupled receptors exclusively activated by designer drugs $\left(\mathrm{G}_{\mathrm{q}^{-}}\right.$ DREADD) and pharmacological approaches were used to test the hypothesis that activation of SN DA neurons and subsequent DS D1 receptor signaling can enhance fear extinction learning. Results suggest that nigrostriatal DA and DS D1 receptors could be a novel target for the prevention of fear relapse after extinction.

\section{MATERIALS AND METHODS}

\section{Animals}

A total of 43 adult, male $\mathrm{Tg}(\mathrm{TH}-\mathrm{Cre}) 3.1 \mathrm{Deis}$, Long-Evans rats were provided by Dr Karl Deisseroth via the National Institutes of Health Rat Resource and Research Center (Columbia, MO; Witten et al, 2011) and 88 adult, male wild-type Long-Evans rats (Charles River, Wilmington, MA) were pair-housed in Nalgene Plexiglass cages $(45.5 \mathrm{~W} \times 24 \mathrm{D} \times 21 \mathrm{H} \mathrm{cm})$, in a temperature- $\left(22^{\circ} \mathrm{C}\right)$ and humidity-controlled vivarium accredited by the Association for Assessment and Accreditation of Laboratory Animal Care located on the University of Colorado Denver Auraria campus. Rats were kept on a $12 \mathrm{~h}$ light-dark cycle with ad libitum access to food and water. Protocols were approved by the University of Colorado Denver institutional animal care and use committee.

\section{Surgical Procedures}

AAV5/hSyn-DIO-hm3D $\left(\mathrm{G}_{\mathrm{q}}\right)$-mCherry ( $\mathrm{G}_{\mathrm{q}}$-DREADD) viral vector (University of North Carolina Gene Therapy Viral Core, Chapel Hill, NC; Zhu and Roth, 2014) was microinfused ( $1 \mu \mathrm{l} /$ hemisphere; $0.1 \mu \mathrm{l} / \mathrm{min}$ ) bilaterally into the SN pars compacta $(-5.4 \mathrm{~mm}$ anterior, $\pm 2.3 \mathrm{~mm}$ lateral, $-8.8 \mathrm{~mm}$ ventral) of $\mathrm{TH}-\mathrm{Cre}$ rats under Ketamine $(75.0 \mathrm{mg} / \mathrm{kg}$ i.p.) and Medetomidine $(0.5 \mathrm{mg} / \mathrm{kg}$ i.p.) anesthesia. Carprofen $(5 \mathrm{mg} / \mathrm{kg}$ s.c.) for pain management and Penicillin G (22 $000 \mathrm{IU} /$ rat, s.c.) were administered at induction and every $24 \mathrm{~h}$ for $72 \mathrm{~h}$ after surgery. Behavioral tests were conducted 1 month after viral injection. Viral transfection in the midbrain was verified in all rats by inspection of mCherry (Olympus BX53; Center Valley, PA). Images through SN projection sites implicated in fear extinction were captured from at least 8 hemispheres per brain region per animal, and intensity of mCherry signal above background (densitometry) was calculated as previously described (Lloyd et al, 2017). Wild-type rats received bilateral 26-gauge guide cannula (4.6 mm below pedestal; Plastics One, Torrington, CT) targeted to the DS $(+5.0 \mathrm{~mm}$ anterior, $+3.0 \mathrm{~mm}$ lateral, $-4.5 \mathrm{~mm}$ ventral; Greenwood et al, 2012; Strong et al, 2011) and recovered for 1 week before behavioral testing.

\section{Drugs}

Clozapine-N-Oxide (CNO; National Institute of Mental Health Chemical Synthesis and Drug Supply Program, Bethesda, MD) was dissolved immediately before use in sterile saline with $0.6 \%$ DMSO (Boekhoudt et al, 2016). Vehicle-treated rats received equal volume of $0.6 \%$ DMSO in saline. Vehicle or CNO ( $1 \mathrm{mg} / \mathrm{kg}$ i.p.) was administered $30 \mathrm{~min}$ before fear extinction training sessions. 2,3,4,5,tetrahydro-7,8-dihydroxy-1-phenyl-1H-3-benzazepine hydrochloride (SKF38393; $0.5 \mu \mathrm{g} / \mu \mathrm{l}$; Agnoli et al, 2013; Sigma-Aldrich, St Louis, MO) and 6-chloro-7,8-dihydroxy-1phenyl-2,3,4,5-tetrahydro-1H-3-benzazepine hydrobromide (SKF81297; $0.1 \mu \mathrm{g} / \mu \mathrm{l}$; Larkin et al, 2016; Tocris Biosciences, Minneapolis, MN) were dissolved in sterile saline and microinjected into the DS $(1 \mu \mathrm{l} /$ hemisphere, rate of $0.5 \mu \mathrm{l} /$ min) through microinjectors extending $0.5 \mathrm{~mm}$ below the guide tip, $10 \mathrm{~min}$ before fear extinction.

\section{Behavior}

Auditory fear conditioning (4 CS-US presentations: $10 \mathrm{~s}$, $80 \mathrm{~dB}, 2 \mathrm{kH}$ auditory CS co-terminating with a $1 \mathrm{~s}, 0.8 \mathrm{~mA}$ foot shock, $1 \mathrm{~min}$ ITI), auditory fear extinction (20 CS; $1 \mathrm{~min}$ ITI), and fear renewal testing (4 CS; 1 min ITI) took place in contexts $\mathrm{A}, \mathrm{B}$, and $\mathrm{C}$, respectively, following protocols used previously (Bouchet et al, 2017; Wang et al, 2016; see Supplementary Methods for details). ABC, as opposed to $\mathrm{ABA}$, renewal was used to limit the contribution of contextual fear memory to fear expression during renewal. Although the use of a novel renewal context in the $\mathrm{ABC}$ renewal paradigm could increase susceptibility to external disinhibition, a novel renewal context was used to maintain consistency with prior work investigating renewal (Maren, 2014; Neumann and Kitlertsirivatana, 2010; Wang et al, 2016; Goode and Maren, 2014).

\section{Double-Label Fluorescent In Situ Hybridization}

At 2 days following the renewal test, a subset of TH-Cre rats were injected i.p. with CNO $(1 \mathrm{mg} / \mathrm{kg})$ or vehicle and, $30 \mathrm{~min}$ later, either exposed to fear extinction or left in their home cages. Brains were processed with fluorescent in situ hybridization (FISH) for D1 and D2 mRNAs, or D1 and cfos mRNAs as previously described (Herrera et al, 2016; Mika et al, 2015). Images (1.7 to $-0.8 \mathrm{~mm}$ Bregma) through the DMS, DLS, NAc core (NAcC), and NAc shell (NAcS) were captured on a confocal fluorescent microscope (200X, Axio Observer Z1; Zeiss Microscopy, Jena, Germany) from 4 distinct hemispheres from at least 2, but up to 4 , brain sections. Single cfos, D1, D2, and double-labeled cells were counted by multiple experimenters blind to treatment conditions using Zeiss Zen software (Herrera et al, 2016; Mika et al, 2015).

\section{Statistical Analyses}

Percent time spent freezing was calculated by averaging freezing data from individual experimenters with immobility times obtained from Noldus Ethovision XT. Pre-shock freezing for each test was averaged and group differences were analyzed with ANOVA. Average freezing across trials during conditioning and extinction were analyzed using repeated-measures ANOVA with subsequent Viral Expression Site or Drug as factors. Freezing across renewal trials was averaged and compared using 2 (Same vs Different) $\times 3$ (DREADD study levels: Veh vs Off-Target CNO vs SN CNO; or D1 agonist study levels: Veh vs SKF38393 vs SKF81297) 
ANOVA. Group differences in cFos protein expression were analyzed with $2 \times 3$ ANOVA. Group differences in total number of $c f o s$ or D1 mRNA-expressing neurons and \% D1expressing neurons containing cfos mRNA were also analyzed with a $2 \times 2$ ANOVA. mCherry density between brain regions was analyzed with ANOVA. Fisher's protected least significant differences post hoc analyses were performed when appropriate. Group differences were considered significant when $p \leqslant 0.05$.

\section{RESULTS}

\section{Activation of SN DA Neurons Enhances Fear Extinction Memory and Blocks Fear Renewal}

Four rats had no visible mCherry and were excluded from analyses. Rats with mCherry expression restricted to at least one full SN hemisphere and no observable mCherry in the VTA were assigned after the experiment to the SN group $(n=16)$. Eight $\mathrm{SN}$ rats received $\mathrm{CNO}(\mathrm{SN} \mathrm{CNO})$ and 8 received vehicle. Rats with minimal mCherry in the $\mathrm{SN}$, but with visible mCherry in the VTA, were assigned to the OffTarget group. mCherry was observed in a portion of at least one hemisphere of the VTA in all 22 of the Off-Target rats. Twelve Off-Target rats were injected with $\mathrm{CNO}$ and 10 were injected with Vehicle. No significant differences were found between SN and Off-Target rats injected with Vehicle, and hence these rats were combined into the 'Vehicle' group $(n=18)$. Figure $1 \mathrm{~b}$ depicts mCherry expression in brain regions of $\mathrm{SN}$ rats. A photomicrograph depicting terminal expression of mCherry in the DS is shown in Figure 1c. Of the mCherry-positive neurons, $92 \%$ were found to coexpress TH immunoreactivity (Figure $1 \mathrm{~d}-\mathrm{f}$ ). Thus, although most of the viral transfection was restricted to DA-containing neurons, contribution of non-DA neurons to behavioral effects cannot be ruled out.

The $\mathrm{G}_{\mathrm{q}}$-DREADD approach allows selective increases in phasic activation of discrete populations of DA neurons when the synthetic ligand $\mathrm{CNO}$ is injected i.p. (Alexander et $a l, 2009)$. To determine the effects of $\mathrm{G}_{\mathrm{q}}$-DREADDinduced SN DA activation during fear extinction on subsequent extinction memory and renewal, rats were injected with either vehicle or CNO during 2 days of fear extinction. Fear conditioning and fear renewal were conducted drug free (Figure 2a shows experimental timeline). Freezing was negligible before the first CS-US presentation during conditioning (Figure $2 \mathrm{~b}$; pre). All rats acquired fear conditioning (Main effect of time: $F(3,105)=16.421$, $p<0.0001$; Figure 2b) and there was no difference in freezing between groups (Main effect of group: $\mathrm{F}(2,25)=2.2 ; p=0.1$ ). The post hoc analyses revealed that the SN CNO group did not differ from the Vehicle group during any of the conditioning trials, and only differed from the Off-Target CNO group during trial $4(p<0.05)$.

All groups displayed within-session fear extinction (Main effect of time: $F(4,140)=55.004, p<0.0001$; Figure $2 c$ ) that was similar between groups (Main effect of group: $F$ $(2,35)=0.6 ; p=0.5)$. Average freezing during the first 2 fear extinction trials was almost identical between groups ( $F$ $(2,35)=0.1 ; p=0.9)$, indicating that the nonsignificant difference in freezing observed during fear conditioning did not result in differential fear memory between groups.
When tested for fear extinction memory, all rats displayed within-session fear extinction (Main effect of time: $F$ $(4,140)=26.45, p<0.0001$; Figure $2 \mathrm{~d})$, but the SN CNO group displayed significantly less freezing than rats in other groups (Main effect of group: $\mathrm{F}(2,35)=3.22, \quad p=0.05$; Figure $2 \mathrm{~d}$ ), indicating enhanced fear extinction recall in rats whose SN DA neurons were activated during fear extinction. The interaction between viral expression and time just missed significance $(\mathrm{F}(8,140)=1.89, p=0.06)$. The post hoc analyses indicated that the SN CNO group differed from the Off-Target CNO group during trials $2-4$ and 14, and from the Vehicle group during trials 4, 9, and 18.

Rats were again exposed to the CS the next day in either the Same or a Different context for assessment of fear renewal. Significant fear renewal was noted in the Vehicle and Off-Target groups (main effect of context; $\mathrm{F}$ $(1,32)=9.20, p=0.005$; Figure 2e), but activation of $\mathrm{SN}$ DA neurons during extinction blocked fear renewal (Group by context interaction: $\mathrm{F}(2,32)=3.35 ; p=0.04$; Figure 2e). Rats whose SN DA neurons were activated during fear extinction displayed altered neural activation patterns in regions important for contextual processing (CA1 of the hippocampus) and driving the fear response (CeA), but not in regions traditionally implicated in fear extinction memory, such as the IL of the PFC (Sotres-Bayon and Quirk, 2010; Knapska et al, 2012; Supplementary Figure S1).

\section{Gq-DREADD Activates Target D1-Expressing Neurons in the DS}

We used double D1/cfos FISH to determine whether the $\mathrm{G}_{\mathrm{q}}$-DREADD approach successfully activates target D1expressing neurons in the DS (see Figure 3a for regions counted). Virtually no colocalization of D1 and D2 mRNAs was observed in the DS (Figure 3b). As almost all neurons in the DS express either D1 or D2 mRNA, cells expressing single cfos mRNA in the DS can be assumed to be putative D2-expressing neurons (Gerfen et al, 1990).

A representative photomicrograph showing D1 and cfos mRNAs in the DMS of a rat exposed to extinction is depicted in Figure 3c. Extinction learning increased the percentage of D1-expressing neurons containing cfos mRNA in the DMS $(\mathrm{F}(1,14)=5.548, p=0.03$; Figure $3 \mathrm{~d})$ and the number of single cfos mRNA-expressing cells in the DLS (F $(1,14)=4.750, p=0.04$; Table 1$)$. Fear extinction learning did not alter the number of single cfos mRNA-containing cells nor the percentage of D1-expressing neurons containing cfos mRNA in the NAcC or NAcS (Table 1), but extinction learning increased the number of neurons expressing D1 mRNA in the NAcC $(\mathrm{F}(1,14)=4.634, p=0.04$; Table 1$)$. Activation of $\mathrm{G}_{\mathrm{q}}$-DREADD with $\mathrm{CNO}$ increased both the number of cells expressing cfos mRNA $(\mathrm{F}(1,14)=4.474$, $p=0.05$; Table 1) and the percentage of D1-expressing neurons containing cfos mRNA in the DMS (F $(1,14)=13.492, p=0.003$; Figure $3 \mathrm{~d})$ and the number of double-labeled D1/cfos mRNA-expressing neurons in the DLS $(F(1,16)=5.564, p=0.03$; Figure $3 \mathrm{~d})$, but had no effect in the NAcC or NAcS (Table 1 and Figure $3 \mathrm{e}$ ). We have previously observed that exposure to a familiar environment does not elicit appreciable Fos products in the striatum (Herrera et al, 2016), and thus the cfos mRNA observed in the extinction group likely reflects an effect of fear extinction. 

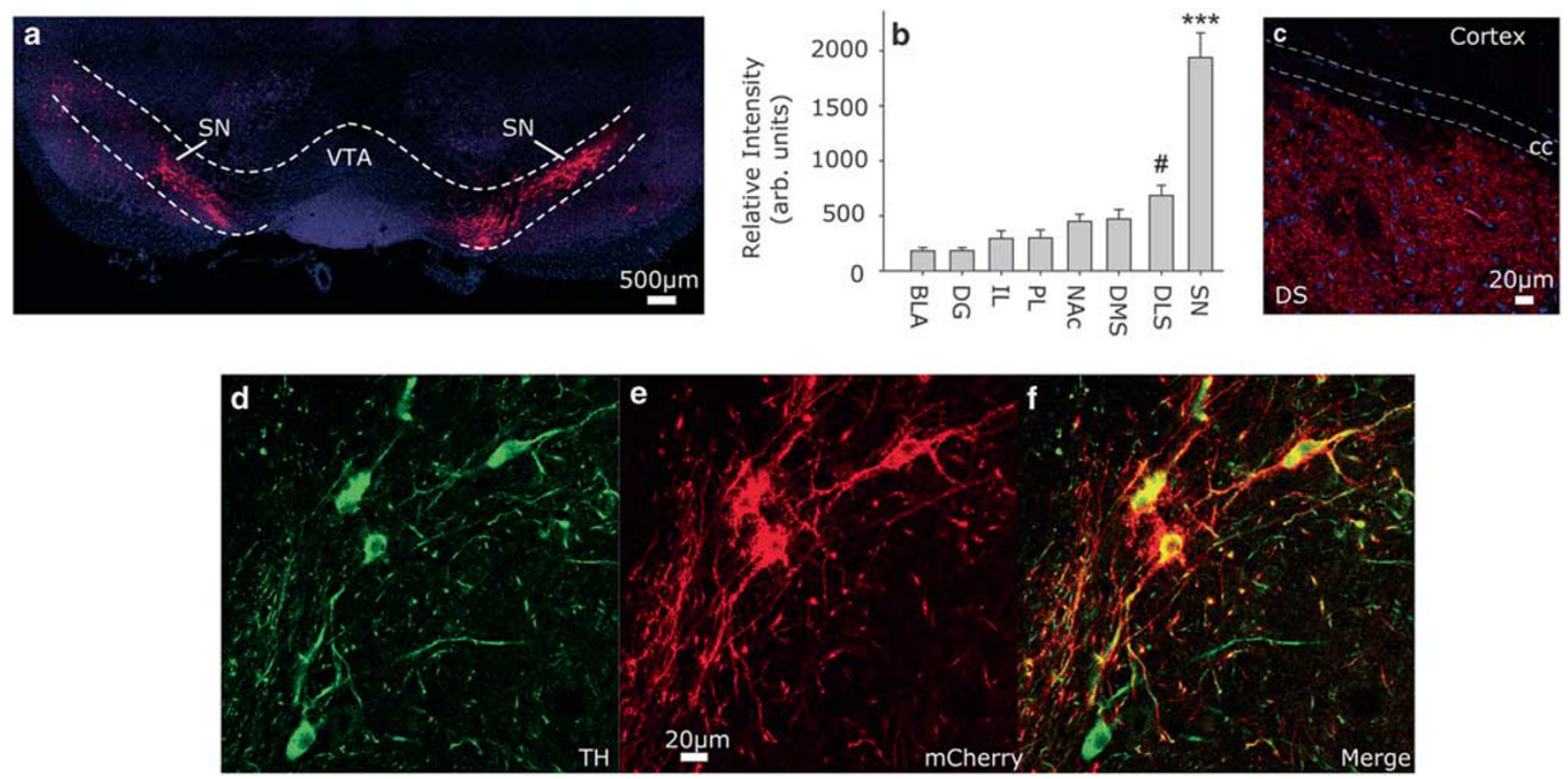

Figure I Expression of viral $G_{q}$-coupled designer receptors exclusively activated by designer drugs ( $G_{q}$-DREADD). (a) Representative photomicrograph depicting bilateral mCherry (red) in the substantia nigra (SN). Blue is 4',6-diamidino-2-phenylindole (DAPI). (b) mCherry intensity in SN and terminal regions including basolateral amygdala (BLA), dentate gyrus (DG), infralimbic cortex (IL), prelimbic cortex (PL), nucleus accumbens (NAc), dorsal medial striatum (DMS), and dorsal lateral striatum (DLS). (c) Representative photomicrograph of terminal mCherry expression in the dorsal striatum (DS). (d) Tyrosine hydroxylase $(\mathrm{TH})$ immunoreactivity in the SN, (e) mCherry autofluorescence in the SN, ( $\mathrm{f}$ ) merge of TH and mCherry. Data presented represent mean \pm SEM. ${ }^{\#} P<0.0$ I relative to $\mathrm{BLA}, \mathrm{DG}, \mathrm{IL}$, and $\mathrm{PL} ;{ }^{*} * * * * 0.000$ I relative to all other groups.

a

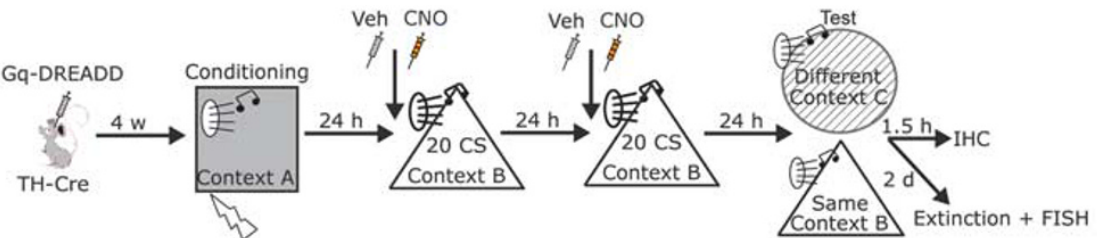

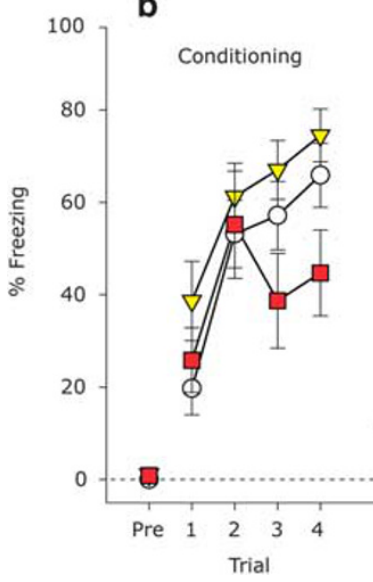

c d Extinction Day 1 $-\mathrm{O}-$ Vehicle

Renewal

Extinction Day 2 Same पIIIC Different $\Phi \rightarrow$ Off-Target CNO
$\rightarrow-$ SN CNO $*$

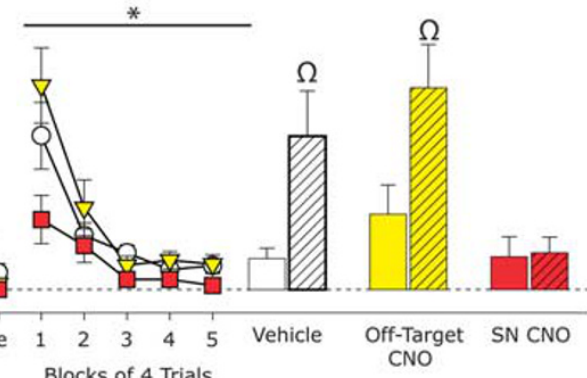

Figure 2 Effect of activation of substantia nigra (SN) dopamine neurons on fear extinction and fear renewal. (a) Experimental design. TH-Cre rats received injections of a viral vector containing a construct coding for a Cre-dependent $\mathrm{G}_{\mathrm{q}}$-coupled designer receptor exclusively activated by a designer drug ( $\mathrm{G}_{\mathrm{q}}$ DREADD). Rats were exposed to auditory fear conditioning, followed by 2 consecutive days of auditory fear extinction training preceded by administration of Vehicle (Veh) or Clozapine-N-Oxide (CNO; I mg/kg). (b) Levels of freezing during fear conditioning. (c,d) Levels of freezing during fear extinction training days. Arrows indicate Veh or CNO administration 30 min before fear extinction. (e) Levels of freezing during the renewal test. Rats in the Vehicle and OffTarget CNO groups exhibited an increase in fear in response to the tone when placed into a novel context (Different) as compared with those placed into the same context as extinction was learned (Same). This fear renewal was absent in the SN CNO group. Data presented represent mean \pm SEM. $* P=0.05$ SN $\mathrm{CNO}$ compared with Vehicle and Off-Target $\mathrm{CNO}$ groups; ${ }^{\Omega} p<0.05$ relative to all other groups except each other. 
Activation of DS D1 Receptors during Fear Extinction Blocks Fear Renewal without Enhancing Fear Extinction Memory

To determine the contribution of D1 receptors in the DS to the observed effects of $\mathrm{G}_{\mathrm{q}}$-DREADD-induced activation of SN DA neurons, D1 agonists were microinjected into the DS before fear extinction learning on 2 subsequent days, and a
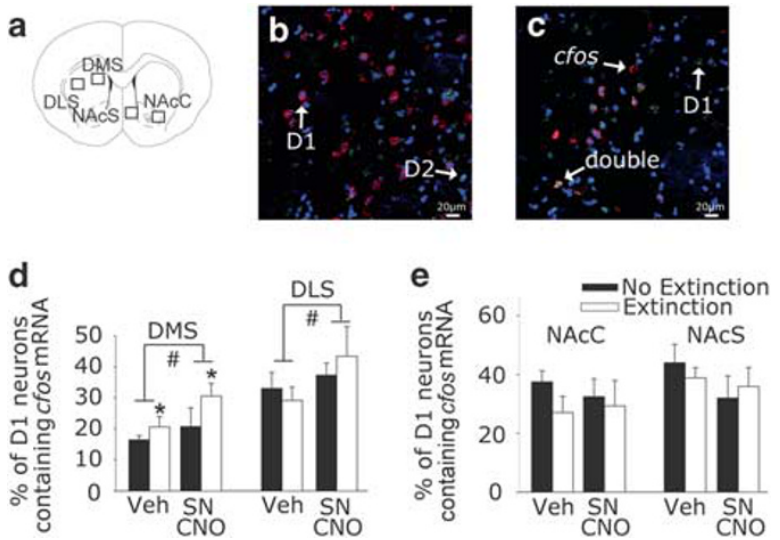

Figure 3 Double fluorescent in situ hybridization (FISH) labeling of cfos and DI mRNAs after fear extinction. TH-Cre rats that received intra-SN injections of a viral vector containing a construct coding for a $G_{q}$-coupled designer receptor exclusively activated by a designer drug were injected with either Vehicle (Veh) or Clozapine-N-Oxide (SN CNO) and then were either left in their home cages (No Extinction) or were exposed to auditory fear extinction (Extinction) 30 min later. (a) Coronal section modified from the Paxinos and Watson rat brain atlas (Paxinos and Watson, 1998) showing the regions counted. (b) Representative photomicrograph from dorsal medial striatum (DMS) showing DI (red) and D2 (green) mRNA expression. Blue is 4',6-diamidino-2-phenylindole (DAPI). (c) Representative photomicrograph from the DMS showing DI (green) and cfos (red) mRNA expression. (d,e) Percent of DI neurons containing cfos mRNA within the DMS, dorsal lateral striatum (DLS), nucleus accumbens core (NAcC), and nucleus accumbens shell (NACS). Data presented represent mean \pm SEM. ${ }^{*} P<0.05$ main effect of fear extinction; ${ }^{\#} p<0.05$ main effect of drug. fear renewal was assessed drug free $24 \mathrm{~h}$ later (Figure $4 \mathrm{a}$ ). Because FISH revealed that the $\mathrm{G}_{\mathrm{q}}$-DREADD approach activates target D1-expressing neurons in both the DMS and DLS, cannulae were targeted between the DMS and DLS. Given reports that systemic administration of SKF81297 and SKF38393 can differentially impact fear extinction (Abraham et al, 2016; Borowski and Kokkinidis, 1998), we microinjected both drugs into the DS in different cohorts of rats before fear extinction. Out of 54 rats, 52 had successful bilateral DS cannulae (Figure 4j), resulting in the following group sizes: Saline Same $=12$, Saline Different $=11$, SKF38393 Same $=8, \quad$ SKF38393 Different $=9, \quad$ SKF81297 Same $=6$, and SKF81297 Different $=6$.

Rats acquired auditory fear conditioning equally (Main effect of time: $F(3,147)=65.679, p<0.0001$; Figure $4 \mathrm{~b}$ ), regardless of subsequent group assignment. Neither agonist affected freezing during the first fear extinction training session, during which all rats displayed within-session extinction (Main effect of time: $F(4,196)=56.950$, $p<0.0001$; Figure 4c). Similar effects were observed during the second fear extinction training session the next day (Main effect of time: $\mathrm{F}(4,196)=46.846, \quad p<0.0001$; Figure $4 \mathrm{~d}$ ). Freezing to the first few CS during a fear extinction session represents memory of previously acquired fear extinction (Do-Monte et al, 2015), thus freezing during the first 4 trials were analyzed separately. Intra-DMS D1 agonist had no impact on fear extinction memory during the first 4 trials of the second fear extinction training session. Similarly, all rats displayed equivalent freezing in the Same context when tested drug free the next day. It is unlikely that the presence of the D1 agonist during the extinction memory test (extinction day 2; Figure 4d) obscured a potential effect of prior D1 agonist on extinction memory, because similar results were observed in a different cohort of rats tested drug free a day after fear extinction in the presence of intra-DS SKF38393 (Supplementary Figure S2).

Despite the observation that D1 activation had no effect on fear extinction learning or memory in the extinction context,

Table I The Effects of CNO Injection and Fear Extinction on DI and cfos mRNA Levels within the Dorsal Medial Striatum (DMS), Dorsal Lateral Striatum (DLS), Nucleus Accumbens Core (NAcC), and Nucleus Accumbens Shell (NAcS)

\begin{tabular}{|c|c|c|c|c|}
\hline Brain region & $\begin{array}{c}\text { Vehicle No Extinction } \\
\text { mean }( \pm \text { SEM) }\end{array}$ & $\begin{array}{l}\text { Vehicle Extinction } \\
\text { mean }( \pm \text { SEM) }\end{array}$ & $\begin{array}{l}\text { CNO No Extinction } \\
\text { mean }( \pm \text { SEM) }\end{array}$ & $\begin{array}{l}\text { CNO Extinction } \\
\text { mean }( \pm \text { SEM })\end{array}$ \\
\hline \multicolumn{5}{|c|}{ No. of single DI mRNA-positive cells } \\
\hline DLS & $28.50(4.67)$ & $27.90(3.82)$ & $23.67(4.04)$ & $29.66(1.34)$ \\
\hline NAcC* & $29.78(2.75)$ & $38.08(6.5 \mathrm{I})$ & $25.49(2.32)$ & $39.79(3.77)$ \\
\hline \multicolumn{5}{|c|}{ No. of single cfos mRNA-positive cells } \\
\hline $\mathrm{DMS}^{\#}$ & $3.28(0.65)$ & $4.2(0.86)$ & $4.57(1.32)$ & $7.03(1.12)$ \\
\hline DLS* & $4.02(0.59)$ & $6.875(1.367)$ & $4.25(1.32)$ & $8.31(2.50)$ \\
\hline $\mathrm{NAcC}$ & $4.65(1.19)$ & $6.24(1.66)$ & $4.36(2.45)$ & $6.04(1.86)$ \\
\hline NAcS & $4.18(1.02)$ & $6.39(1.39)$ & $4.86(1.84)$ & $7.37(0.72)$ \\
\hline
\end{tabular}

Rats received either Vehicle or CNO injections, then were either exposed to fear extinction (Extinction) or left in their home cage (No Extinction) for 30 min. FISH analysis was conducted to quantify levels of DI and cfos mRNA.

*Main effect of extinction $(p<0.05)$.

\#Main effect of drug $(p=0.05)$. 
a
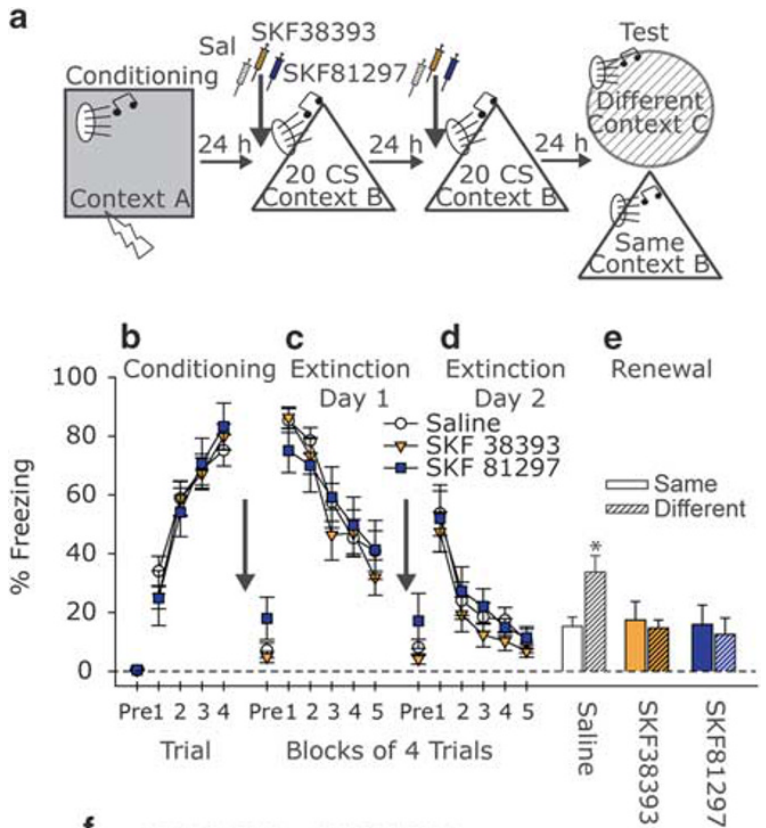

f

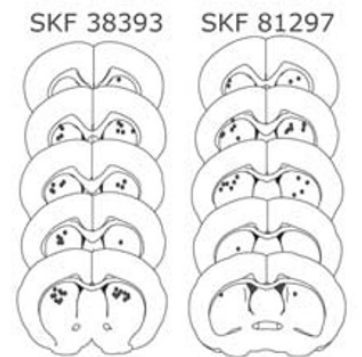

Figure 4 The effects of DI agonists in the DS during fear extinction on fear extinction memory and fear renewal. (a) Experimental design. Rats received injections of Saline or DI agonists (SKF 38393 or SKF 8| 297; I $\mu$ // hemisphere) into the DS before fear extinction learning, and then were placed drug free into either the familiar fear extinction context (Same) or a novel context (Different) to test for fear renewal. (b) Levels of freezing during auditory fear conditioning. (c,d) Levels of freezing during two sequential days of auditory fear extinction training in the presence of either Saline or DI agonist. (e) Levels of freezing during the fear renewal test. ( $f$ ) Black dots represent location of cannulae tips in the dorsal striatum. Data presented represent mean \pm SEM. ${ }^{*} P<0.05$ compared with all other groups.

rats that received D1 agonist before fear extinction were protected against fear renewal tested in a novel context $24 \mathrm{~h}$ after the second fear extinction training session (Drug by context interaction: $\mathrm{F}(2,46)=3.1, p<0.0001$; Figure $4 \mathrm{e})$. The post hoc tests revealed that the Saline Different rats froze more than all other groups, and neither the SKF38393 Different nor SKF81297 Different groups differed from their Same counterparts (Figure $4 \mathrm{e}$ ).

\section{DISCUSSION}

Data presented here reveal a novel role for nigrostriatal DA in fear extinction. Activation of SN DA neurons with $\mathrm{G}_{\mathrm{q}^{-}}$ DREADD during fear extinction had no effect on fear extinction acquisition, but enhanced fear extinction memory and blocked the renewal of fear; a pattern of data paralleled by cFos expression in the CeA. D1-expressing neurons in the DS are a likely target mediating at least part of the effect of
SN DA activation. Indeed, expression of terminal mCherry, indicating presence of $\mathrm{G}_{\mathrm{q}}$-DREADD, was highest in the DS, D1-expressing neurons in the DMS were observed to be recruited during fear extinction, and $G_{\mathrm{q}}$-DREADD-induced DA potentiated activity of D1-expressing neurons in both the DMS and the DLS. Interestingly, rats whose SN DA neurons were activated by $\mathrm{G}_{\mathrm{q}}$-DREADD during prior fear extinction displayed potentiated cFos expression in the CA1 of the hippocampus, a region important for contextual processing. Pharmacological activation of DS D1 receptors during fear extinction had no impact on fear extinction, but blocked fear renewal in a novel context. Together, these data suggest that activation of SN DA neurons and DS D1 receptors could render fear extinction memory resistant to memorydisrupting effects of changes in context, thus preventing fear renewal.

Systemic manipulations that enhance DA signaling can enhance fear extinction and reduce relapse (Haaker et al, 2013; Abraham et al, 2014), but prior work investigating the roles of specific DA circuits in fear extinction reveal inconsistent results. VTA DA neurons projecting to the PFC can impair fear extinction (Hitora-Imamura et al, 2015); however, other studies implicate involvement of D1 and D2 in the PFC in fear extinction (Hikind and Maroun, 2008; Mueller et al, 2010). Midbrain DA neurons also provide DA innervation to other limbic structures including the BLA, hippocampus, and NAc. Although recent work elucidates a role for the BLA-NaC pathway in fear extinction (Correia et al, 2016), DA manipulations in the BLA have no effect on fear extinction (Fiorenza et al, 2012; Hikind and Maroun, 2008). In contrast, D1 activation in the hippocampus can enhance short-term fear extinction memory (Fiorenza et al, 2012), whereas inhibition of D2 in the NAc has been reported to impair fear extinction (Holtzman-Assif et al, 2010). Notably, relapse was not investigated in these studies; therefore, the effects of these manipulation on fear renewal is unknown. No studies to our knowledge have investigated the involvement of SN DA neurons and their primary target, the DS, in fear extinction.

Here we used a $\mathrm{G}_{\mathrm{q}}$-DREADD approach in $\mathrm{TH}$-Cre rats to selectively increase phasic activity of SN DA neurons (Zhu and Roth, 2014). This approach was selective at targeting DA neurons in the SN (Figure 1) and produced a functional increase in activity of target DS D1-expressing neurons downstream of SN DA neurons (Figure 3d). The effects of SN DA activation were not because of a nonspecific effect of DA on locomotor activity. Indeed, $\mathrm{CNO}$ did not alter general locomotor activity measured by spontaneous cage crossings in Context $\mathrm{B}$ before the first CS during either fear extinction training session (Supplementary Table S1), nor did CNO reduce freezing during the first extinction training day. These observations suggest that the reduced freezing displayed by the SN CNO group during extinction training day 2 was not due to an effect of $\mathrm{CNO}$ on the expression of freezing per se, but rather to SN DA facilitating the later recall of fear extinction. The effects of SN DA activation were also unlikely to be due to differential fear conditioning between groups. Indeed, freezing levels up to at least $84 \%$ can be detected during conditioning under these conditions (Supplementary Figure S2), indicating that the lack of statistical difference between the SN CNO and Vehicle groups during conditioning was not due to a ceiling 
effect. Moreover, no group differences in fear memory were observed during the first few trials of extinction training day 1.

A critical question is where DA is acting to produce the observed effects of SN DA activation. Although the SN projects to regions other than just the DS, minimal terminal mCherry was observed in regions traditionally implicated in fear extinction, such as the PFC, hippocampus, and AMG. Therefore, it is unlikely that the observed effects of SN DA activation were mediated by DA in these regions, although a role for DA in these regions cannot be completely ruled out. Double FISH revealed that D1-expressing neurons in the DMS are recruited during fear extinction learning (Figure 3d). DMS-cortical circuits are involved in guiding goal-directed behavior (Shiflett et al, 2010) that may increase as the animal acquires extinction and begins to evaluate the environment. We have observed potentiated activity of DMS D1-expressing neurons during recall of relapse-resistant fear extinction in rats that ran in wheels during fear extinction (Mika et al, 2015). Together, these data suggest that DMS D1-expressing neurons could represent a previously unidentified component of fear extinction, recruitment of which could be involved in the learning or recall of fear extinction. In addition, although DLS D1-expressing neurons were not recruited during fear extinction, $\mathrm{G}_{\mathrm{q}}$-DREADD-induced $\mathrm{SN}$ DA activity resulted in increased cfos in these DLS neurons (Figure 3d). The DLS is involved in guiding inflexible behavioral strategies that can occur at the expense of goaldirected or spatial strategies (ie, 'habit'; Schwabe et al, 2008; Yin and Knowlton, 2006). These data therefore raise the possibility that DA in the DLS elicited by $\mathrm{G}_{\mathrm{q}}$-DREADD could have altered the learning strategy used during fear extinction acquisition to one involving the DLS. This is an intriguing possibility, as learning strategies involving the DLS may be less susceptible to the disruptive effects of contingency changes, such as context, during recall (Schwabe et al, 2008). More selective targeting of DMS vs DLS DA circuits will be required to determine the role of DA in these regions in fear extinction. Similarly, how these DS regions communicate with canonical fear circuitry is currently unknown. The observation of altered cFos expression patterns during fear extinction memory recall in the CeA and CA1 (Supplementary Figure S1d and e) implicates these regions in the circuit through which nigrostriatal DA enhances fear extinction.

D1 agonists injected into the DS before fear extinction prevented fear renewal without affecting extinction memory (Figure 4e), even when extinction memory was assessed drug free (Supplementary Figure S2); a pattern of data differing from that of SN DA activation. This discrepancy could be explained by phasic DA elicited by $\mathrm{G}_{\mathrm{q}}$-DREADD interacting with DA receptors differently than receptor agonists. For example, phasic DA activates both D1- and D2-expressing neurons in the DMS (Table 1), whereas the partial D1 agonist SKF38393 is selective to D1 (Conroy et al, 2015). SKF81297, however, agonizes both D1 and D2 receptors (Rashid et al, 2007). Another possibility is that DA in the DS could render extinction memories resistant to renewal, whereas $\mathrm{DA}$ in a different $\mathrm{SN}$ target could be responsible for enhancing extinction memory. As D1 agonists were targeted to the middle of the DS and the drugs likely spread to both the DMS and DLS, the effects of pharmacological D1 activation cannot shed light on where within the $D S G_{q^{-}}$
DREADD-induced DA could have been acting. However, the pharmacological data do support the idea that nigrostriatal DA reduces fear renewal through a mechanism involving D1 receptors in the DS.

The current data demonstrate for the first time that augmenting activity of SN DA neurons can facilitation fear extinction memory. Importantly, fear extinction supported by nigrostriatal DA or DS D1 signaling is resistant to fear renewal. As relapse phenomena, including renewal, are a barrier to the long-term remission of anxiety and traumarelated disorders, the nigrostriatal DA pathway provides a promising target for the development of more effective therapeutic strategies.

\section{FUNDING AND DISCLOSURE}

Dr. Greenwood received funding for these studies from the NIH (R15MH114026) and a University of Colorado Denver Office of Resource Services faculty development grant. All other authors declare no conflict of interest.

\section{ACKNOWLEDGMENTS}

We thank Dr Michael V Baratta and Dr Erik B Oleson for technical advice on the use of DREADDs.

\section{REFERENCES}

Abraham AD, Neve KA, Lattal KM (2014). Dopamine and extinction: a convergence of theory with fear and reward circuitry. Neurobiol Learn Mem 108: 65-77.

Abraham AD, Neve KA, Lattal KM (2016). Activation of D1/5 dopamine receptors: a common mechanism for enhancing extinction of fear and reward-seeking behaviors. Neuropsychopharmacology 41: 2072-2081.

Agnoli L, Mainolfi P, Invernizzi RW, Carli M (2013). Dopamine D1-like and D2-like receptors in the dorsal striatum control different aspects of attentional performance in the five-choice serial reaction time task under a condition of increased activity of corticostriatal inputs. Neuropsychopharmacology 38: 701-714.

Alexander GM, Rogan SC, Abbas AI, Armbruster BN, Pei Y, Allen JA et al (2009). Remote control of neuronal activity in transgenic mice expressing evolved G protein-coupled receptors. Neuron 63: 27-39.

Badrinarayan A, Wescott SA, Vander Weele CM, Saunders BT, Couturier BE, Maren S et al (2012). Aversive stimuli differentially modulate real-time dopamine transmission dynamics within the nucleus accumbens core and shell. J Neurosci 32: 15779-15790.

Boekhoudt L, Omrani A, Luijendijk MC, Wolterink-Donselaar IG, Wijbrans EC, van der Plasse G et al (2016). Chemogenetic activation of dopamine neurons in the ventral tegmental area, but not substantia nigra, induces hyperactivity in rats. Eur Neuropsychopharmacol 26: 1784-1793.

Borowski TB, Kokkinidis L (1998). The effects of cocaine, amphetamine, and the dopamine D1 receptor agonist SKF 38393 on fear extinction as measured with potentiated startle: implications for psychomotor stimulant psychosis. Behav Neurosci 112: 952-965.

Bouchet CA, Lloyd BA, Loetz EC, Farmer CE, Ostrovskyy M, Haddad $\mathrm{N}$ et al (2017). Acute exercise enhances the consolidation of fear extinction memory and reduces conditioned fear relapse in a sex-dependent manner. Learn Mem 24: 358-368.

Bouton ME, Ricker ST (1994). Renewal of extinguished responding in a 2nd context. Anim Learn Behav 22: 317-324. 
Chen W, Wang Y, Wang X, Li H (2017). Neural circuits involved in the renewal of extinguished fear. IUBMB Life 69: 470-478.

Conroy JL, Free RB, Sibley DR (2015). Identification of G proteinbiased agonists that fail to recruit beta-arrestin or promote internalization of the D1 dopamine receptor. ACS Chem Neurosci 6: 681-692.

Correia SS, McGrath AG, Lee A, Graybiel AM, Goosens KA (2016). Amygdala-ventral striatum circuit activation decreases longterm fear. Elife 5: e12669.

Do-Monte FH, Manzano-Nieves G, Quinones-Laracuente K, Ramos-Medina L, Quirk GJ (2015). Revisiting the role of infralimbic cortex in fear extinction with optogenetics. J Neurosci 35: 3607-3615.

Dreyer JK, Herrik KF, Berg RW, Hounsgaard JD (2010). Influence of phasic and tonic dopamine release on receptor activation. $J$ Neurosci 30: 14273-14283.

Fiorenza NG, Rosa J, Izquierdo I, Myskiw JC (2012). Modulation of the extinction of two different fear-motivated tasks in three distinct brain areas. Behav Brain Res 232: 210-216.

Fitzgerald PJ, Seemann JR, Maren S (2014). Can fear extinction be enhanced? A review of pharmacological and behavioral findings. Brain Res Bull 105: 46-60.

Gerfen CR, Engber TM, Mahan LC, Susel Z, Chase TN, Monsma FJ Jr. et al (1990). D1 and D2 dopamine receptor-regulated gene expression of striatonigral and striatopallidal neurons. Science 250: 1429-1432.

Goode TD, Maren S (2014). Animal models of fear relapse. ILAR J 55: 246-258.

Greenwood BN, Strong PV, Loughridge AB, Day HE, Clark PJ, Mika A et al (2012). 5-HT2C receptors in the basolateral amygdala and dorsal striatum are a novel target for the anxiolytic and antidepressant effects of exercise. PLoS ONE 7: e46118.

Haaker J, Gaburro S, Sah A, Gartmann N, Lonsdorf TB, Meier K et al (2013). Single dose of L-dopa makes extinction memories context-independent and prevents the return of fear. Proc Natl Acad Sci USA 110: E2428-E2436.

Herrera JJ, Fedynska S, Ghasem PR, Wieman T, Clark PJ, Gray N et al (2016). Neurochemical and behavioural indices of exercise reward are independent of exercise controllability. Eur J Neurosci 43: 1190-1202.

Hikind N, Maroun M (2008). Microinfusion of the D1 receptor antagonist, SCH23390 into the IL but not the BLA impairs consolidation of extinction of auditory fear conditioning. Neurobiol Learn Mem 90: 217-222.

Hitora-Imamura N, Miura Y, Teshirogi C, Ikegaya Y, Matsuki N, Nomura H (2015). Prefrontal dopamine regulates fear reinstatement through the downregulation of extinction circuits. Elife 4: e08274.

Holtzman-Assif O, Laurent V, Westbrook RF (2010). Blockade of dopamine activity in the nucleus accumbens impairs learning extinction of conditioned fear. Learn Mem 17: 71-75.

Howe MW, Tierney PL, Sandberg SG, Phillips PE, Graybiel AM (2013). Prolonged dopamine signalling in striatum signals proximity and value of distant rewards. Nature 500: 575-579.

Knapska E, Macias M, Mikosz M, Nowak A, Owczarek D, Wawrzyniak $M$ et al (2012). Functional anatomy of neural circuits regulating fear and extinction. Proc Natl Acad Sci USA 109: 17093-17098.

Knapska E, Maren S (2009). Reciprocal patterns of c-Fos expression in the medial prefrontal cortex and amygdala after extinction and renewal of conditioned fear. Learn Mem 16: 486-493.

Kravitz AV, Kreitzer AC (2012a). Striatal mechanisms underlying movement, reinforcement, and punishment. Physiology (Bethesda) 27: 167-177.
Kravitz AV, Tye LD, Kreitzer AC (2012b). Distinct roles for direct and indirect pathway striatal neurons in reinforcement. Nat Neurosci 15: 816-818.

Larkin JD, Jenni NL, Floresco SB (2016). Modulation of risk/reward decision making by dopaminergic transmission within the basolateral amygdala. Psychopharmacology (Berl) 233: 121-136.

Lenz JD, Lobo MK (2013). Optogenetic insights into striatal function and behavior. Behav Brain Res 255: 44-54.

Lloyd BA, Hake HS, Ishiwata T, Farmer CE, Loetz EC, Fleshner M et al (2017). Exercise increases mTOR signaling in brain regions involved in cognition and emotional behavior. Behav Brain Res 323: $56-67$.

Maren S (2014). Fear of the unexpected: hippocampus mediates novelty-induced return of extinguished fear in rats. Neurobiol Learn Mem 108: 88-95.

Mika A, Bouchet CA, Bunker P, Hellwinkel JE, Spence KG, Day HE et al (2015). Voluntary exercise during extinction of auditory fear conditioning reduces the relapse of fear associated with potentiated activity of striatal direct pathway neurons. Neurobiol Learn Mem 125: 224-235.

Mueller D, Bravo-Rivera C, Quirk GJ (2010). Infralimbic D2 receptors are necessary for fear extinction and extinction-related tone responses. Biol Psychiatry 68: 1055-1060.

Neumann DL, Kitlertsirivatana E (2010). Exposure to a novel context after extinction causes a renewal of extinguished conditioned responses: implications for the treatment of fear. Behav Res Ther 48: 565-570.

Paxinos G, Watson C (1998). The Rat Brain in Stereotaxic Coordinates. Academic Press: New York.

Rashid AJ, O'Dowd BF, Verma V, George SR (2007). Neuronal Gq/ 11-coupled dopamine receptors: an uncharted role for dopamine. Trends Pharmacol Sci 28: 551-555.

Schultz W (2016). Dopamine reward prediction error coding. Dialogues Clin Neurosci 18: 23-32.

Schwabe L, Dalm S, Schachinger H, Oitzl MS (2008). Chronic stress modulates the use of spatial and stimulus-response learning strategies in mice and man. Neurobiol Learn Mem 90: 495-503.

Shiflett MW, Brown RA, Balleine BW (2010). Acquisition and performance of goal-directed instrumental actions depends on ERK signaling in distinct regions of dorsal striatum in rats. $J$ Neurosci 30: 2951-2959.

Sotres-Bayon F, Quirk GJ (2010). Prefrontal control of fear: more than just extinction. Curr Opin Neurobiol 20: 231-235.

Strong PV, Christianson JP, Loughridge AB, Amat J, Maier SF, Fleshner $\mathrm{M}$ et al (2011). 5-hydroxytryptamine 2C receptors in the dorsal striatum mediate stress-induced interference with negatively reinforced instrumental escape behavior. Neuroscience 197: 132-144.

Tronson NC, Corcoran KA, Jovasevic V, Radulovic J (2012). Fear conditioning and extinction: emotional states encoded by distinct signaling pathways. Trends Neurosci 35: 145-155.

Wang Q, Jin J, Maren S (2016). Renewal of extinguished fear activates ventral hippocampal neurons projecting to the prelimbic and infralimbic cortices in rats. Neurobiol Learn Mem 134(Pt A): 38-43.

Wise RA (2009). Roles for nigrostriatal-not just mesocorticolimbicdopamine in reward and addiction. Trends Neurosci 32: 517-524.

Witten IB, Steinberg EE, Lee SY, Davidson TJ, Zalocusky KA, Brodsky $\mathrm{M}$ et al (2011). Recombinase-driver rat lines: tools, techniques, and optogenetic application to dopamine-mediated reinforcement. Neuron 72: 721-733.

Yin HH, Knowlton BJ (2006). The role of the basal ganglia in habit formation. Nat Rev Neurosci 7: 464-476.

Zhu H, Roth BL (2014). DREADD: a chemogenetic GPCR signaling platform. Int J Neuropsychopharmacol 18: pii: pyu007.

Supplementary Information accompanies the paper on the Neuropsychopharmacology website (http://www.nature.com/npp) 